\title{
An Integrated Plasmo-Photoelectronic Nanostructure Biosensor Detects an Infection Biomarker Accompanying Cell Death in Neutrophils
}

\author{
Younggeun Park, Byunghoon Ryu, Qiufang Deng, Baihong Pan, Yujing Song, Yuzi Tian, \\ Hasan B. Alam, Yongqing Li,* Xiaogan Liang,* and Katsuo Kurabayashi*
}

Bacterial infections leading to sepsis are a major cause of deaths in the intensive care unit. Unfortunately, no effective methods are available to capture the early onset of infectious sepsis near the patient with both speed and sensitivity required for timely clinical treatment. To fill the gap, the authors develop a highly miniaturized $\left(2.5 \times 2.5 \mu \mathrm{m}^{2}\right)$ plasmo-photoelectronic nanostructure device that detected citrullinated histone $\mathrm{H} 3$ (CitH3), a biomarker released to the blood circulatory system by neutrophils. Rapidly detecting $\mathrm{CitH} 3$ with high sensitivity has the great potential to prevent infections from developing life-threatening septic shock. To this end, the author's device incorporates structurally engineered arrayed hemispherical gold nanoparticles that are functionalized with highaffinity antibodies. A nanoplasmonic resonance shift induces a photoconduction increase in a few-layer molybdenum disulfide $\left(\mathrm{MoS}_{2}\right)$ channel, and it provides the sensor signal. The device achieves label-free detection of serum CitH3 with a 5-log dynamic range from $10^{-4}$ to $10^{7} \mathrm{ng} \mathrm{mL}$ and a sample-to-answer time $<20 \mathrm{~min}$. Using this biosensor, the authors longitudinally measure the dynamic CitH3 profiles of individual living mice in a sepsis model at high resolution over 12 hours. The developed biosensor may be poised for future translation to personalized management of systemic bacterial infections.

\section{Introduction}

The human society has experienced a number of illnesses caused by bacterial infections throughout its entire history. As a potentially life-threatening form of infectious illness, sepsis causes more than 250000 annual deaths in the United States alone. ${ }^{[1]}$ Despite significant efforts made for sepsis diagnosis, existing methods still suffer major shortcomings, including a long sampleto-answer time, low sensitivity, and insufficient selectivity. For example, microbial culture to identify the source of infection, which is one of the gold standards of sepsis diagnosis, may take over 48 hours for incubation, and false negative results (over 24\%) are very common due to a low density of blood bacteria at the early stage of infection. ${ }^{[2]}$ Without timely diagnosis and treatment, the condition can quickly deteriorate and result in septic shock that causes multiple organ dysfunction syndrome (MODS) or mortality accompanied by severe systemic inflammation (Figure 1a). A therapeutic strategy guided by a biomarker accurately reflecting the presence and severity of the disease is a promising approach for sepsis management. ${ }^{[3,4]}$

Neutrophils are essential immune cells to combat microbial infections in the front line of defense. ${ }^{[5]}$ One of the defense mechanisms of neutrophils involves cell death induced by the release of neutrophil extracellular traps (NETs), which form a meshwork of chromatin fibers mixed with granule-derived antimicrobial
Dr. Y. Park, B. Ryu, Y. Song, Prof. X. Liang, Prof. K. Kurabayashi

Department of Mechanical Engineering

University of Michigan

Ann Arbor, MI, 48109 USA

E-mail: xiaoganl@umich.edu; katsuo@umich.edu

Dr. Y. Park, Prof. Y. Li, Prof. X. Liang, Prof. K. Kurabayashi

Center for Integrative Research in Critical Care

University of Michigan

Ann Arbor, MI, 48109 USA

E-mail:yqli@med.umich.edu

Dr. Q. Deng, Dr. B. Pan, Prof. H. B. Alam, Prof. Y. Li

Department of Surgery

University of Michigan

Ann Arbor, MI, 48109 USA

\author{
Dr. Q. Deng \\ Department of Endocrinology and Metabolism \\ Third Xiangya Hospital of Central South University \\ Changsha, Hunan 410013, China \\ Dr. B. Pan, Y. Tian \\ Department of General Surgery \\ The Xiangya Hospital of Central South University \\ Changsha, Hunan 410008, China \\ Y. Tian \\ Department of Rheumatology \\ The Xiangya Hospital of Central South University \\ Changsha, Hunan 410008, China
}

The ORCID identification number(s) for the author(s) of this article can be found under https://doi.org/10.1002/smll.201905611.

DOI: 10.1002/smll.201905611 
a
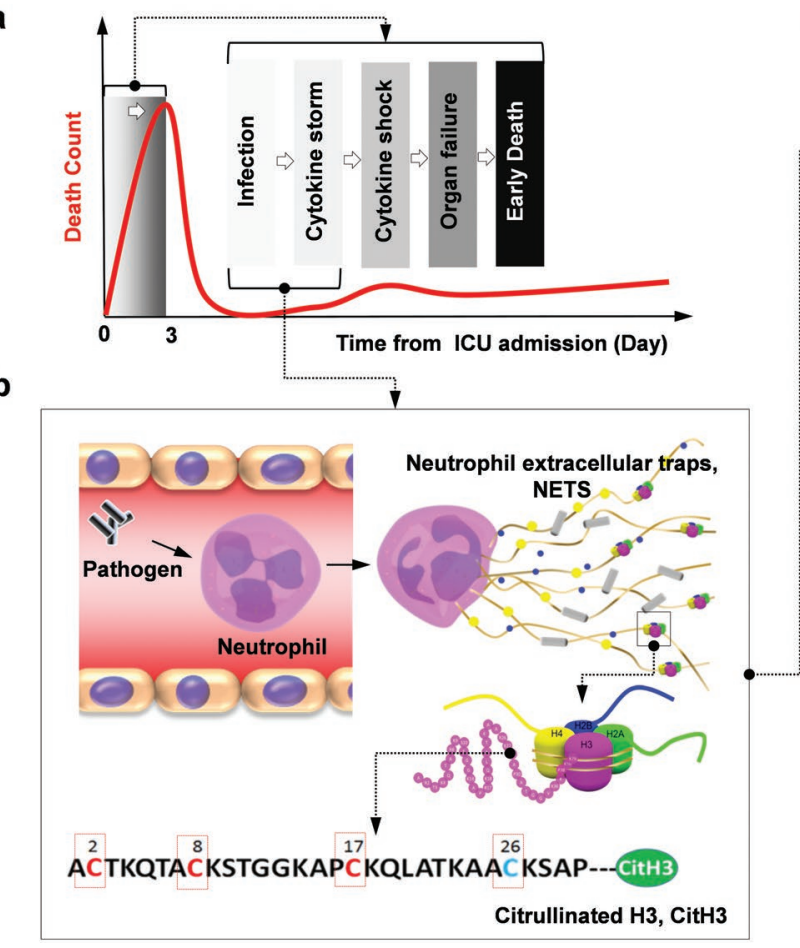

C

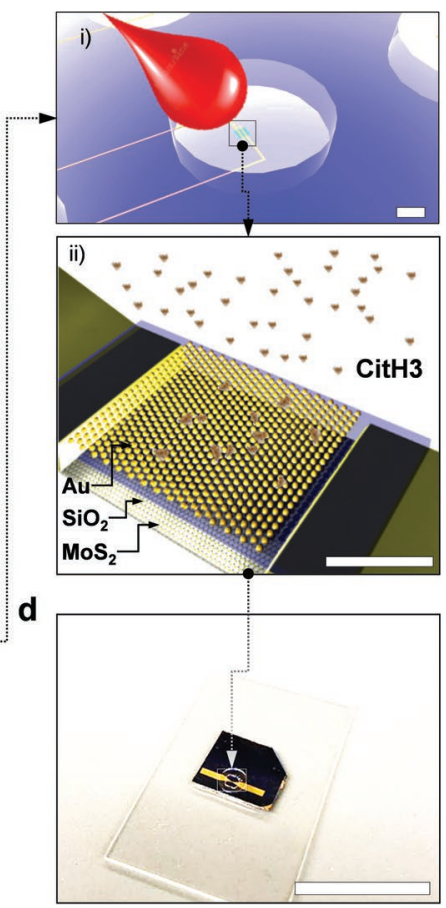

Figure 1. Neutrophil extracellular traps (NETs)-induced sepsis biomarker detected by integrated nano optoelectronic biosensor (iNOBS). a) Death count of patients in septic shock after intensive care unit (ICU) admission. b) CitH3 released into blood circulation upon neutrophil extracellular trap (NET)-induced immune cell death (NETosis) occurring in response to severe infection. CitH3 serves as a specific sepsis biomarker at an early stage of sepsis development. The 4 unique citrullinated molecular sites (4 Cits) of $\mathrm{CitH} 3$ are used for high-affinity binding with the custom-made antibody in this study. c) Schematic of the iNOBS device architecture and operation; i) loading of a sample (e.g., serum) of small volume ( $\approx 2.5 \mu \mathrm{L})$ onto a polydimethylsiloxane (PDMS) microfluidic well (scale bar $=200 \mu \mathrm{m})$, ii) settling of biomarker molecules onto the device surface $(\mathrm{scale}$ bar $=500 \mathrm{~nm})$. d) Photo image of the iNOBS chip (scale bar $=15 \mathrm{~mm}$ ).

peptides and enzymes to kill bacteria extracellularly. Citrullination of histone $\mathrm{H} 3$ (CitH3), catalyzed by peptidylarginine deiminase (PAD), has recently been identified to be involved in an early step in the NETs-induced cell death (NETosis) in response to severe infection (Figure $1 \mathrm{~b}$ ). ${ }^{[6]}$ Serum levels of CitH3 are significantly elevated with increasing severity of lipopolysaccharide (LPS)-induced septic shock. ${ }^{[7]}$ Circulating $\mathrm{CitH} 3$ can be detected early and serve as a selective biomarker of septic shock, and administering a drug that diminishes or abolishes circulating $\mathrm{CitH} 3$ improves the survival of septic mice. ${ }^{[8]}$ As such, $\mathrm{CitH} 3$ is a more promising biomarker to provide an early sign of septic shock and guide therapy than other biomarkers widely used to monitor severe infection and pro-inflammatory responses, such as procalcitonin (PCT), interleukin (IL-6), and IL-1 $\beta \cdot{ }^{[7,8]}$ However, timely CitH3-guided sepsis management is prohibited by the lack of a technology enabling quick, sensitive, and accurate detection of the $\mathrm{CitH} 3$ biomarker at the point of care (POC).

Researchers have extensively explored POC biomarker analysis techniques using localized surface plasmon resonance (LSPR) sensors incorporating antibody-conjugated metallic nanoparticles. ${ }^{[9,10]}$ These LSPR biosensors are advantageous for POC measurements ${ }^{[11-13]}$ as they are label-free, robust, rapid, costeffective, and easy to integrate into miniaturized fluidic devices with simple optics. ${ }^{[14-16]}$ They are also attractive for diagnosing and monitoring the trajectory of critically ill patients. ${ }^{[16,17]}$ Our previous study ${ }^{18]}$ demonstrated that strategically integrating a
LSPR biosensing layer above a molybdenum disulfide $\left(\mathrm{MoS}_{2}\right)$ photoconductive channel allows for constructing a self-contained photoelectronic protein biosensor. The small device architecture of this biosensor eliminates the need for large-volume off-chip detectors in signal transduction, such as charge-coupled devices (CCDs) and ultrahigh-resolution photospectrometers, thus potentially serving as a POC platform for $\mathrm{CitH} 3$-based early infection diagnosis. Unfortunately, this device still faces two major issues; (i) it cannot achieve sufficiently fast speed because of the significantly weak affinity of $\mathrm{CitH} 3$ to antibodies on the commercial market; and (ii) it falls short of reaching the highest sensitivity in $\mathrm{CitH} 3$ detection due to the unoptimized structural arrangement of gold nanoparticles on its LSPR biosensing layer.

Here, we report a highly miniature $\left(2.5 \times 2.5 \mu \mathrm{m}^{2}\right)$ biosensor device combining nanoscale plasmonic and photoelectronic effects for detection of the $\mathrm{CitH} 3$ infection biomarker (Figure 1c,d). In this study, we constructed structurally optimized hemispherical gold nanoparticle arrays, namely "gold nanohemispheres (AuNHs)" (Figure 2a), using a highly scalable self-assembly material synthesis technique. After functionalizing AuNHs with our lab-made high-affinity $\mathrm{CitH} 3$ monoclonal antibodies, ${ }^{[8]}$ we incorporated them into the aforementioned LSPR-MoS ${ }_{2}$ device architecture. ${ }^{[18]}$ The device was newly termed the "integrated nano optoelectronic biosensor (iNOBS)" and was shown to achieve optoelectronic detection of $\mathrm{CitH} 3$ and other sepsis-related biomarkers (IL-1 $\beta$, and PCT) in serum at high sensitivity. The operation of iNOBS was 
supported by the broadly ranged photoresponsivity of a few-layer $\mathrm{MoS}_{2}$ photoconductive channel. The AuNH surfaces of iNOBS achieved a large electric field enhancement for incident light at a biologically behind near-infrared (NIR) wavelength of $\lambda=650 \mathrm{~nm}$. Taken together, the iNOBS-based label-free binding assay enabled CitH3 detection with a LOD of $0.87 \mathrm{pg} \mathrm{mL}^{-1}$ (56 fM), which is 250-fold smaller than that of commercial ELISA, a large dynamic range of $10^{5}$, a short sample-to-answer time of $20 \mathrm{~min}$, and a small sample volume of $2.5 \mu \mathrm{L}$. The accuracy of our iNOBS-based CitH3 measurement was confirmed by ELISA over a wide range from $10^{-2}$ to $10^{1} \mathrm{ng} \mathrm{mL}^{-1}$. With the remarkable label-free biosensor, we established the ability of continuously monitoring the time-varying CitH3 biomarker profiles in the circulation system of a sepsis mouse model.

\section{Results and Discussions}

\subsection{Plasmo-Photoelectronic Nanostructure and Characterization}

The iNOBS device architecture has three main components: (i) a bio-tunable nanoplasmonic optical filter (Bio-NOF) layer, which is a thin $(100 \mu \mathrm{m}) \mathrm{SiO}_{2}$ substrate covered by gold nanoparticles (AuNPs); (ii) a few-layer $\mathrm{MoS}_{2}$ photoconductive channel underneath the Bio-NOF layer; and (iii) a polydimethylsiloxane (PDMS) layer with a microfluidic well above the Bio-NOF layer. Only a small fraction of incident light transmits through the Bio-NOF layer initially and reaches the $\mathrm{MoS}_{2}$ photoconductive channel, which yields a small baseline photocurrent signal $\left(I_{p h-0}\right)$ (Figure 2a). The resonant behavior of the conduction band electron oscillations near the surface of the optically excited nanoparticles (i.e., LSPR effect) varies with a local refractive index $\left(n_{m}\right)$ change brought by the surface binding of protein molecules.

As illustrated in Figure 2b, constructing highly packed, uniform AuNPs on the Bio-NOF layer is necessary to achieve high sensitivity in CitH3 measurement. A uniform distribution of AuNPs results in a narrow LSPR spectrum curve. Our previous study ${ }^{[18]}$ detected IL-1 $\beta$, a pro-inflammatory cytokine, using a similar device to iNOBS. The AuNPs on the Bio-NOF layer in this device were deposited by a simple, cost-effective method of casting a droplet of colloidally suspended nanoparticles (i.e., drop-casting method). The arrangement of the drop castingdeposited AuNPs showed poor uniformity. ${ }^{[18]}$ However, this was still acceptable in the previous study because strong antigenantibody affinity was found for IL- $1 \beta$. In contract, we expect that CitH3 measurement with weak antibody affinity inherently limits a local refractive index shift in the presence of the analyte at a given concentraion. To address this issue, we structurally engineered self-assembled AuNH arrays with an optimal interparticle distance and density to maximize the sensitivity of our measurement in this study (Figure S1, Supporting Information).

Figure 2c shows a scanning electron microscope (SEM) image of the constructed AuNHs with highly enhanced uniformity and density. The arrayed AuNH arrangement has a surface density of $D_{A u N H} \approx 800$ particles $\mu \mathrm{m}^{-2}$, a particle size of $d_{A u N H}=$ $42 \mathrm{~nm} \pm 10 \%$, and an interparticle distance of $l_{A u N H} \approx 43 \mathrm{~nm}$ with a standard deviation less than $30 \%$ (Figure 2d). Compared to the AuNP arrangement prepared by the drop-casting method, the arrayed $\mathrm{AuNH}$ arrangement showed a five times narrower distribution in both particle size and interparticle distance. Our finite element analysis predicted a strong electric field confined between two neighboring $\mathrm{AuNH}$ particles (Figure S2, Supporting Information). We measured LSPR spectra for both AuNP and AuNH structures with similar values of particle density $\left(\approx 800\right.$ particles $\left.\mu \mathrm{m}^{-2}\right)$ (Figure $2 \mathrm{e}$ ). The spectrum of the arrayed $\mathrm{AuNH}$ arrangement yielded a narrower LSPR shape with a peak at $\lambda=650 \mathrm{~nm}$. We measured the base photocurrent $I_{p h-0}$ for two iNOBS devices, one with a Bio-NOF layer incorporating the drop casting-deposited AuNPs and the other with a Bio-NOF layer incorporating the arrayed AuNHs. Under laser light illumination at $\lambda=650 \mathrm{~nm}$, we observed $I_{p h-0}=0.77 \mu \mathrm{A}$ and $I_{p h-0}=0.1 \mu \mathrm{A}$ at an voltage applied across the $\mathrm{MoS}_{2}$ channel (a drain-source voltage) of $V_{d s}=1 \mathrm{~V}$ for the former (AuNP) and latter (AuNH) devices, respectively (Figure 2f). The significantly smaller $I_{p h-0}$ value for the AuNH device indicates that our self-assembly-based structural engineering approach served well for constructing a Bio-NOF layer with much higher nanoplasmonic optical filtration efficiency.

We fabricated the photoconductive few-layer $\mathrm{MoS}_{2}$ channel on a thermally oxidized silicon substrate using our micro-printing method (Figure 3a and Figure S3, Supporting Information). ${ }^{[19]} \mathrm{A}$ pair of Ti/Au pads laterally sandwiching the $\mathrm{MoS}_{2}$ channel served as the electrode contacts for reading sensor signals (drain current, $\left.I_{d s}\right)$ at $V_{d s}$ (Figure $S 4$, Supporting Information). The effective channel length was measured to be $\approx 1 \mu \mathrm{m}$, which ensured a highly uniform crystal structure for the $\mathrm{MoS}_{2}$ sensing region. The as-printed $\mathrm{MoS}_{2}$ channel had a uniform thickness of $14.5 \mathrm{~nm}$ with $\pm 5 \%$ tolerance along the path that was determined by atomic force microscopy (AFM) (Figure 3b and Figure S5, Supporting Information). This allowed us to operate the iNOBS device with the sufficiently high photoresponsivity of the few-layer $\mathrm{MoS}_{2}$ channel. The laser illumination a $\lambda=650 \mathrm{~nm}$ resulted in 100 times photoenhancement (Figure S6, Supporting Information).

We characterized the LSPR shift of the "bare" arrayed AuNHs prior to antibody conjugation in response to the changing $n_{m}$ of a surrounding medium (glycol-water solution). Additionally, we measured the photoresponsivity $\left(\mathrm{R}=I_{p h} / P_{i n}\right.$, where $P_{i n}$ is power density of incident light.) spectrum of the $\mathrm{MoS}_{2}$ channel (Figure $3 \mathrm{c}$ and Table S1, Supporting Information). The value of $\mathrm{n}_{\mathrm{m}}$ was varied with the glycol-to-water ratio in the medium (see Experimental Section). We observed a reasonably good overlap between the spectra of the photoresponsivity of the $\mathrm{MoS}_{2}$ channel and of the LSPR of the arrayed AuNHs, and the illumination optical band $(\lambda=650 \mathrm{~nm} \pm 10 \%)$. Using an iNOBS device with the bare AuNHs, we measured the variation of $I_{p h}$ with $n_{m}$ and compared it with the LSPR peak shift $\left(\Delta \lambda_{\max }\right)$ characterized above (Figure $3 \mathrm{~d}$ ). The measured $I_{p h}$ linearly correlates to $\Delta \lambda_{\text {max }}\left(\mathrm{R}^{2}=0.9986\right)$, which indeed confirmed that LSPR-shift was responsible for the iNOBS photocurrent signal change (Table S2, Supporting Information). We also provide a comparison of LSPR structures in Table S3, Supporting Information.

\subsection{High-performance Infection Biomarker Detection}

Our study functionalized the AuNHs of our device with a new CitH3 monoclonal antibody (Figure 4 and see Experimental Section). Commercially available CitH3 monoclonal antibodies bind to histone $\mathrm{H} 3$ only at N-terminal with 3 citrullinated sites 


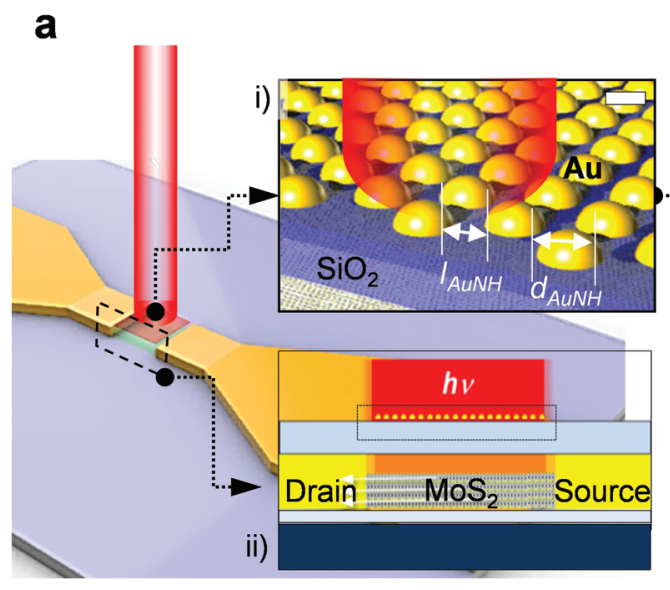

b
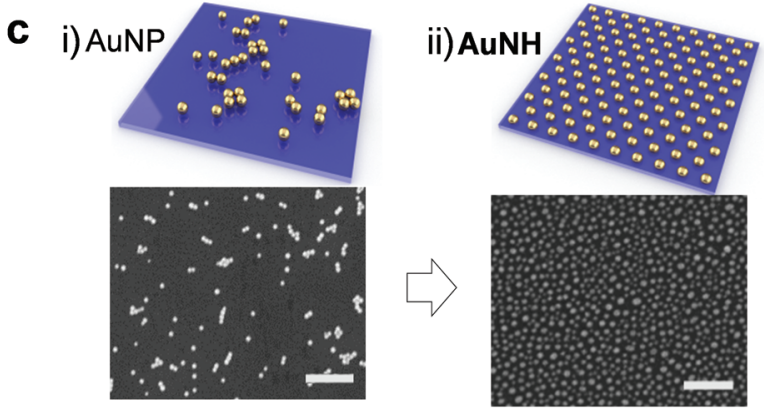

i) Density : High Au Uniformity: High a a a a a a $\mathrm{MoS}_{2}$ ii)

ii) Density : High Uniformity: Low
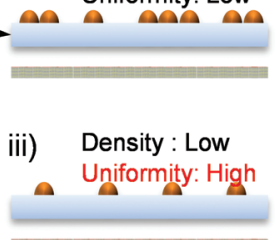
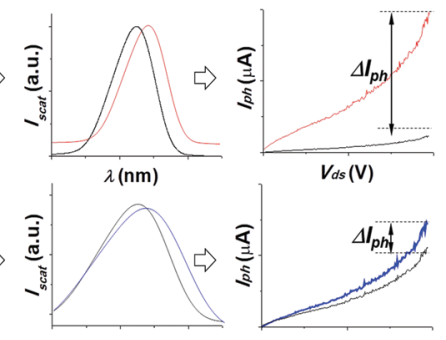

$\lambda(\mathrm{nm})$

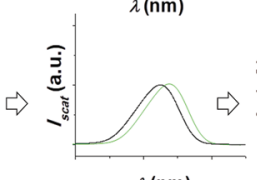

$\lambda(\mathrm{nm})$

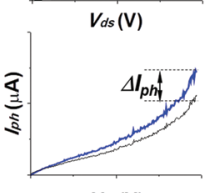

$V d s(\mathrm{~V})$

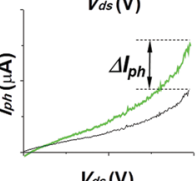

$V_{d s}(\mathbf{V})$ e

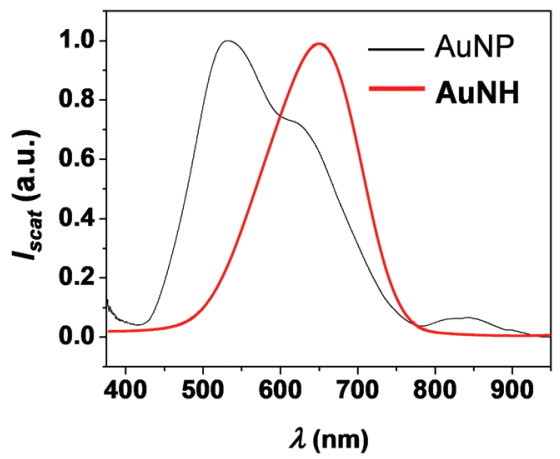

f

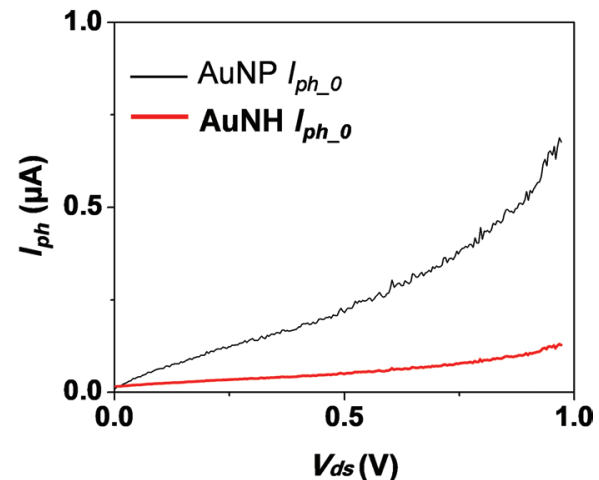

Figure 2. Bio-tunable nanoplasmonic optical filter (Bio-NOF). a) i) Bio-NOF consisting of anti-antibody-conjugated gold nano-hemispheres (AuNHs) on a thin $\mathrm{SiO}_{2}$ substrate (scale bar $=40 \mathrm{~nm}$ ), ii) cross-sectional schematic of the iNOBS device architecture. b) Conceptual illustration showing the impact of the density and uniformity of the gold nanoparticle arrangement on the LSPR spectrum of Bio-NOF and the $I_{p h}-V_{d s}$ curve of iNOBS. i) highdensity, high-uniformity arrangement expected to yield a sharp LSPR spectrum curve and a larger photocurrent increase $\left(\Delta I_{p h}=I_{p h}-I_{p h-0}\right)$ in response to a local refractive index change. ii) high-density, low-uniformity arrangement expected to yield a broad LSPR spectrum curve and a smaller value of $\Delta I_{p h}$. iii) low-density, high-uniformity arrangement expected to yield weak LSPR-based photoabsorption and a smaller value of $\Delta I_{\text {ph }}$. c) Highly scalable self-assembly of optimally engineered gold nanostructural arrays; schematics and scanning electron microscopy (SEM) images of i) drop castingdeposited AuNPs, ii) self-assembled arrayed AuNHs (scale bar $=500 \mathrm{~nm}$ ), d) Analyzed interparticle distance $\left(I_{\text {AuNH }}\right)$ and size distribution $\left(d_{\text {AuNH }}\right)$ of arrayed AuNHs. e) LSPR spectra of drop casting-deposited AuNPs and the arrayedAuNHs. f) $I_{p h}-V_{d s}$ curves for the baseline photocurrent $I_{p h \_0}$ of iNOBS with drop casting-deposited AuNPs and arrayed AuNHs of similar particle density $\left(\approx 800\right.$ particles $\left.\mu \mathrm{m}^{2}\right)$.

(3 Cits) catalyzed by PAD4 alone. This results in limited antibody affinity. In contrast, our antibody was designed in our lab and synthesized in a custom-made manner so that it can tightly interact with 4 unique molecular sites (4 Cits) citrullinated by PAD4 and PAD2 (Figure 1b). Thus, the novel CitH3 antibody has a higher affinity, in addition to specificity, compared to conventional antibodies. Additionally, our recent study shows that our anti-CitH3 antibody can significantly improve survival in a mouse model of sepsis. ${ }^{[20]}$

The iNOBS device enabled simpler and more miniaturized biomarker detection as compared to a conventional LSPR biosensing setup (Figure 4a) (see Experimental Section). 

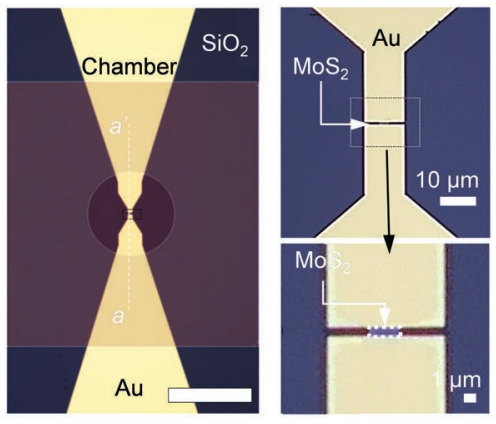

C

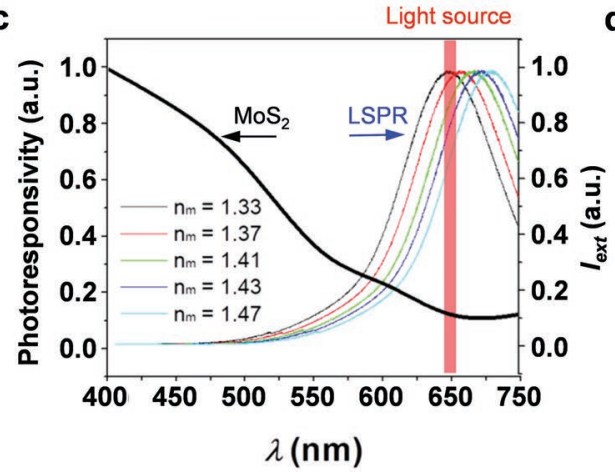

b

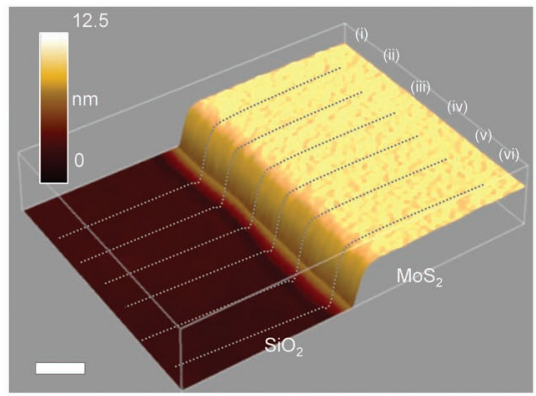

d

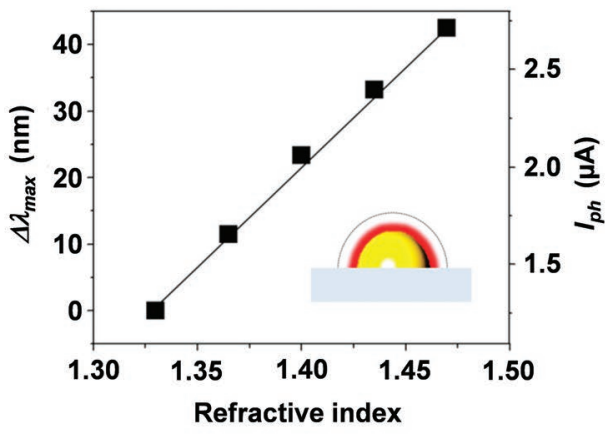

Figure 3. Integrated plasmonic nanostructure array and $\mathrm{MoS}_{2}$ channel. a) Optical micrographs of a $14 \mathrm{~nm}$ thick, $1 \mu \mathrm{m}$ long MoS $\mathrm{S}_{2}$ channel with electrodes (scale bar $=10 \mu \mathrm{m})$. b) Atomic force microscope image of the $\mathrm{MoS}_{2}$ layer on a $\mathrm{SiO}_{2}$ layer (100 nm thickness) (Cross-sectional profiles along the white lines from (i) to (vi) are shown in Figure S5, Supporting Information). c) LSPR spectra of the Bio-NOF with the arrayed AuNHs (arbitrary unit) for the different values of the environmental (bulk) refractive index $\left(n_{m}\right)$ varying from 1.33 to 1.47 and photoresponsivity spectrum of the MoS ${ }_{2}$ photoconductive channel, both compared to the spectral band of the incident light $(\lambda \approx 650 \mathrm{~nm})$. d) LSPR peak wavelength shift $(\Delta \lambda)$ of the arrayed AuNHs versus $I_{p h}$ of the $\mathrm{MoS}_{2}$ photoconductive channel at $V_{d s}=1.0 \mathrm{~V}$ as a function of $n_{m}$.

Before conducting the iNOBS assay illustrated in Figure 4b, we first verified the performance of the Bio-NOF layer for CitH3 quantification using a commercial photo spectrometer (Experimental Section). In this test, we loaded a CitH3-spiked serum sample onto the Bio-NOF layer with $C_{\mathrm{CitH} 3}$ varying from $10^{-4}$ to $10^{1} \mathrm{ng} \mathrm{mL}^{-1}$ (Figure S7, Supporting Information). We observed a total peak wavelength shift of $\Delta \lambda_{\text {max }} \approx$ $10 \mathrm{~nm}$ when $C_{\mathrm{CitH} 3}$ decreased from $10^{1}$ to $10^{-2} \mathrm{ng} \mathrm{mL}{ }^{-1}$. This setup detected no noticeable peak wavelength shift for $C_{\mathrm{CitH} 3}$ $<10^{-2} \mathrm{ng} \mathrm{mL} \mathrm{m}^{-1}$. With our observation here, we confirmed that the optical transmission of the Bio-NOF layer was tuned with the LSPR spectral shift accompanying the variation of $C_{\mathrm{CitH} 3}$.

Figure $4 \mathrm{c}$ plots transient curves of the iNOBS photocurrent signal $\Delta I_{p h}(t)=I_{p h}(t)-I_{p h 0}$ as functions of the incubation time $t$ measured at $V_{d s}=1.0 \mathrm{~V}$ for $C_{\mathrm{CitH} 3}$ between $10^{-4}$ and $10^{1} \mathrm{ng} \mathrm{mL}^{-1}$ (the curve at $C_{\mathrm{CitH}_{3}}=0$ is the baseline). With $C_{\mathrm{CitH} 3}$ reaching the lower $\left(10^{-4} \mathrm{ng} \mathrm{mL} \mathrm{mL}^{-1}\right)$ and upper $\left(10^{1} \mathrm{ng} \mathrm{mL}^{-1}\right)$ bounds, the $\Delta I_{\text {ph }}$ value saturated at $\approx 1.2 \mu \mathrm{A}$ and $\approx 1.7 \mu \mathrm{A}$, respectively, within 20 minutes after loading a serum sample. The $C_{C i t H 3}$ value in a given sample can be quantified from the normalized photocurrent variation $\Delta I_{p h} / I_{p h 0}$. Figure $4 \mathrm{~d}$ shows the standard calibration curve of the iNOBS device with $C_{\mathrm{CitH} 3}$ varying from $10^{-4}$ to $10^{1} \mathrm{ng} \mathrm{mL}^{-1}$. The curve has a sigmoidal shape that is typically observed when antibody-antigen binding reactions are quantified at the full dynamic range. ${ }^{[21]}$ The weak signal at the low concentration range becomes buried in the background noise. The binding sites on the sensor surface becomes saturated with the analyte molecules at the high concentration range. Thus, these two extreme ranges result in the lower and upper saturations of the standard curve. From this curve, we found that the device achieved a LOD of $0.87 \mathrm{pg} \mathrm{mL}^{-1}$ with a large $\left(10^{5}\right)$ dynamic range. The curve also exhibits a trend similar to that obtained from the LSPR peak position $\left(\lambda_{L S P R}\right)$ measured by the LSPR biosensing setup using the commercial photo spectrometer in Figure S7, Supporting Information. While this LSPR biosensing setup failed to distinguish the peak shift for $C_{\mathrm{CitH} 3}<10^{-2} \mathrm{ng} \mathrm{mL} \mathrm{m}^{-1}$ owing to its limited spectral resolution $(\approx 0.1 \mathrm{~nm})$, the iNOBS device was still able to capture a noticeable change of $I_{p h}$ resulting from the small LSPR peak shift even at $C_{\mathrm{CitH} 3}<10^{-2} \mathrm{ng} \mathrm{mL}^{-1}$.

\subsection{Validation of the iNOBS Assay Using Clinical Samples}

Using the iNOBS device, we performed sepsis biomarker analysis for serum samples extracted from living mice (Figure 5). A regression line for correlating the results from iNOBS and commercial ELISA assays measuring $\mathrm{CitH} 3$ was obtained for the sample samples (Figure 5a and Methods). The correlation shows a strong linear relationship $\left(\mathrm{R}^{2}=0.9968\right)$. The iNOBS assay achieved a $\mathrm{LOD}_{\text {iNOBS }}$ as small as $0.87 \mathrm{pg} \mathrm{mL}^{-1}$ whereas the ELISA exhibited an LOD ELISA of $232 \mathrm{pg} \mathrm{mL}^{-1}$ (Table S4, Supporting Information). Here, the ELISA test employed a commercial CitH3 monoclonal antibody (Cayman Chemical, 


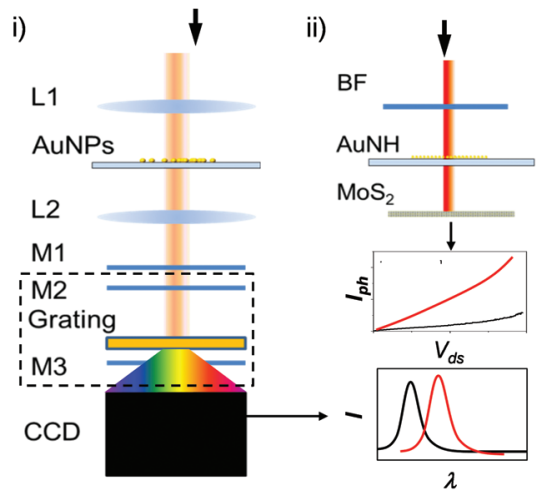

C

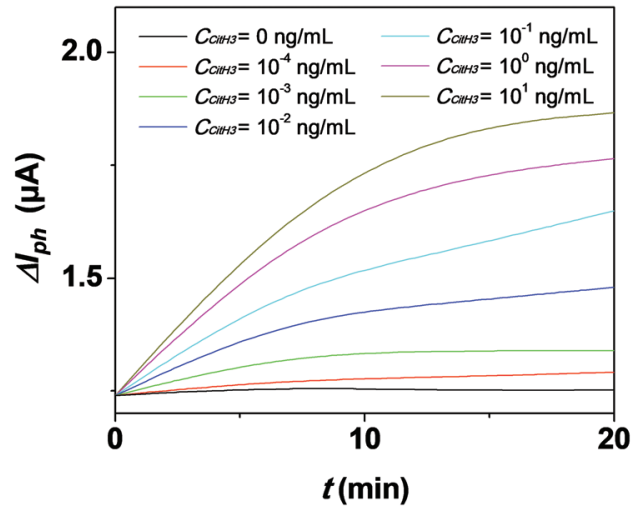

b

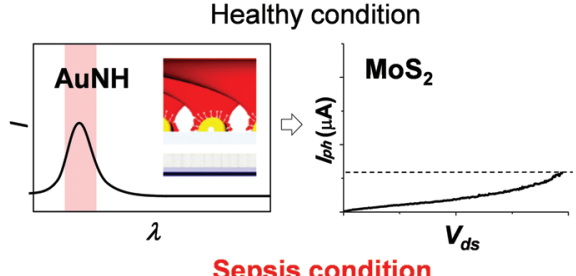

Sepsis condition
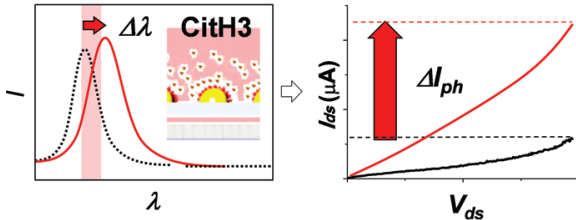

d

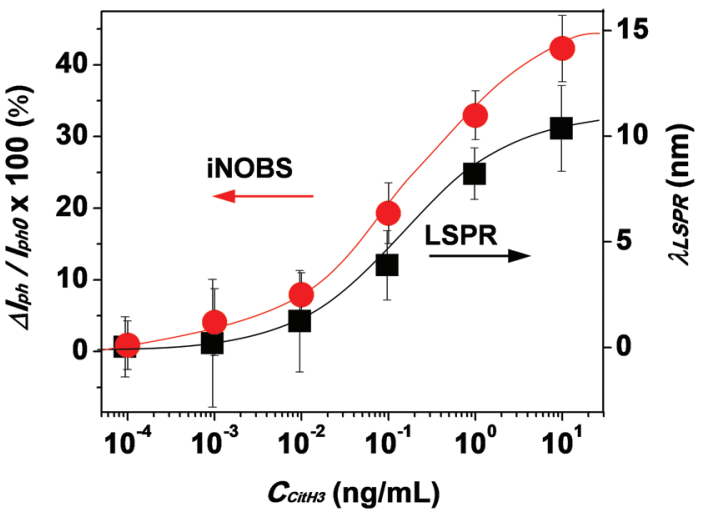

Figure 4. Nano-plasmo-photoelectronic detection performance of iNOBS. a) Setups of i) LSPR spectroscopy measurement (L1: darkfield condenser, L2: objective lens, M1-3: mirror 1-3, CCD: charge-coupled detector, dashed line box: spectrometer) and ii) iNOBS measurement based on LSPR-MoS 2 nano-plasmo-photoelectronic signal transduction (BF: band path filter). b) Binding of $\mathrm{CitH}_{3}$ antigen molecules onto the antibody-conjugated AuNHs continues to decrease the LSPR-induced absorption of incident light at the Bio-NOF layer until the system reaches equilibrium. This is translated to a photocurrent increase $\Delta I_{p h}$ in the $M_{o} S_{2}$ channel over time. c) $\Delta I_{p h}$ at $V_{d s}=1.0 \mathrm{~V}$ as a function of the incubation time after sample loading with $C_{C_{i t H 3}}$ ranging from $10^{-4}$ to $10^{1} \mathrm{ng} \mathrm{mL}^{-1}$. d) Standard calibration curves (photocurrent change $\left(\Delta I_{p h} / I_{p h \_} 0\right)$ versus $\left.C_{C_{\text {itH } 3}}\right)$ obtained using the iNOBS $\left(\right.$ LSPR-MoS ${ }_{2}$ ) device and the LSPR spectroscopy setup.

Ann Arbor, MI, USA). Such a comparison shows that the iNOBS device has a superior concentration sensitivity (or LOD) for $\mathrm{CitH} 3$ over 250 times higher than that of the ELISA assay.

Subsequently, we performed a control experiment to test the iNOBS's selectivity to the target biomarker, i.e., CitH3, in comparison with other four different background biomarkers relevant to sepsis (i.e., PCT, IL- $1 \beta$, IL-6, and TNF- $\alpha$ ) in mouse serum with their concentrations ranging from $10^{-4}$ to $10^{1} \mathrm{ng} \mathrm{mL}^{-1}$ all together (Figure $5 \mathrm{~b}$ ). In this test, we observed no noticeable changes in $\Delta I_{p h} / I_{p h o}$ with respect to the concentrations of any biomarkers other than CitH3. This result indicates negligible cross-reactivity among these biomarkers and therefore proves the capability of the iNOBS assay. Our device enabled highly selective detection of target biomarker molecules.

Furthermore, we applied the iNOBS device in the measurement of circulating $\mathrm{CitH} 3$ in a living mouse treated by cecal ligation and puncture (CLP)-induced sepsis. ${ }^{[22,23]}$ The CLP procedure mimics a ruptured appendix or perforated diverticulitis in human and allows for identifying an irreversible stage of sepsis where the excision of necrotic tissue cannot improve survival. ${ }^{[24,25]}$ Therefore, CLP has been recognized as a standard animal model for sepsis study (Figure 5c). ${ }^{[26]}$ Based on previous studies reporting that mortality was dramatically changed within $24 \mathrm{~h},{ }^{[8,27]}$ we measured $\mathrm{CitH} 3$ for serum samples collected from the mouse at 0,12 , and $24 \mathrm{~h}$ after the CLP procedure (Experimental Section). For comparison, a western blot was also performed for the same mouse serum samples. The serum collected at $0 \mathrm{~h}$ did not show any signature of CitH3 in both the iNOBS assay and the western blot measurement. The CitH3 level in serum reached $\approx 0.11 \mathrm{ng} \mathrm{mL}^{-1}$ at $12 \mathrm{~h}$ after the CLP insult, and further increased to $\approx 0.89 \mathrm{ng} \mathrm{mL}^{-1}$ at $24 \mathrm{~h}$. The western blot images qualitatively matched the iNOBS assay data. Importantly, our iNOBS assay provided consistent quantitative data with a 100-fold faster measurement speed in comparison with the conventional western blot technique.

\subsection{Multi-time Point Measurement of Infection Biomarker Profile for Living Mice}

Finally, we demonstrated iNOBS-based multi-time-point detection of biomarkers using different mouse models (Figure 6). We first obtained time-series profiles of $\mathrm{CitH} 3$ 
a

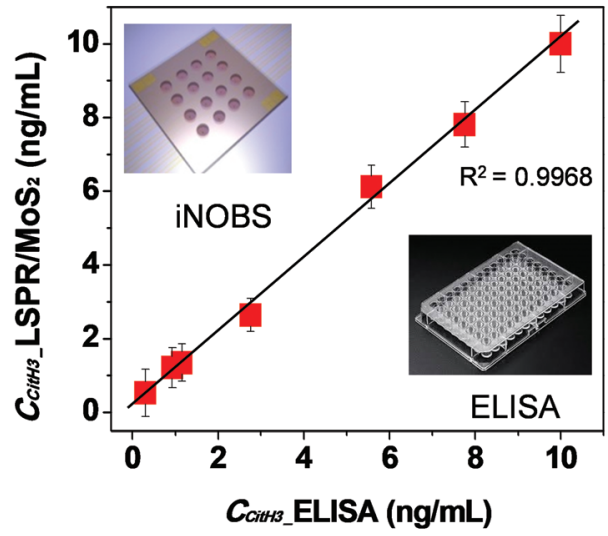

b

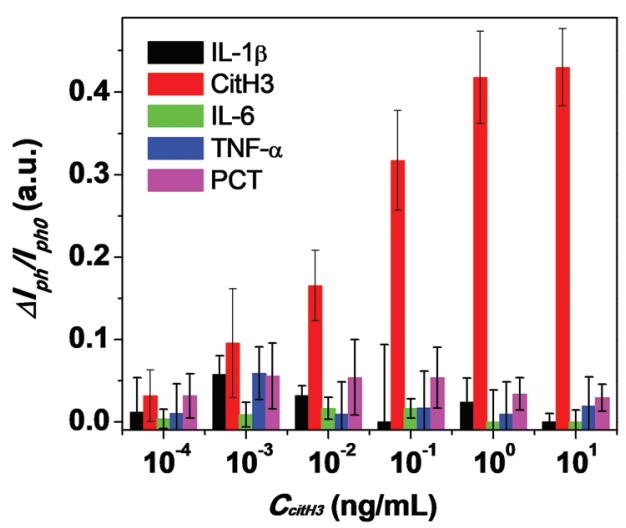

C

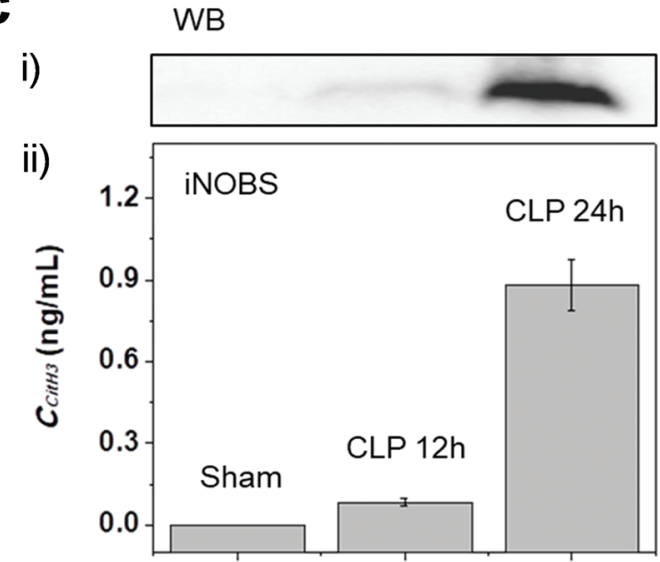

Figure 5. iNOBS-based sensitive and selective sepsis biomarker detection using mouse models. a) Linear regression between iNOBS assay and ELISA data for the same serum samples spiked with $\mathrm{CitH} 3$ at $\mathrm{C}_{\mathrm{CitH} 3}$. ranging from $10^{-2}$ to $10 \mathrm{ng} \mathrm{mL}$. The iNOBS assay was performed $\approx 20$-times faster and 250-times more sensitive than ELISA. b) Photocurrent signal $\left(\Delta l_{p h} / I_{p h o}\right)$ data of the iNOBS obtained for a mixture of IL-1 $\beta$, CitH3, IL-6, TNF- $\alpha$, and PCT in healthy (sham) mouse serum at various concentrations $(n=6)$. The concentrations for the background biomarkers: IL-1 $\beta$; IL-6; TNF- $\alpha$; and PCT were varied so that their values were identical with that of $\mathrm{CitH} 3$ for each measurement. The data show high selectivity of the device to $\mathrm{CitH} 3$ as the signal for $\mathrm{CitH} 3$ is distinctly higher than those for the other biomarkers with a statistical significance $(p \leq 0.01)$. c) CLP mouse model study. CLP treated mouse as a "gold standard clinical sepsis model" which mimics human sepsis development. Comparison of circulating $\mathrm{CitH} 3$ detected by i) western blot and ii) the iNOBS assay at 0,12 and $24 \mathrm{~h}$ after CLP treatment. and two other biomarkers (IL-1 $\beta$ and PCT) for a LPS-injected living mouse over $12 \mathrm{~h}$, at which the animal died (Figure 6a). The measurements were repeated with multiple devices $(\mathrm{n}=5)$. After the injection of LPS at $\approx 1.2 \mathrm{ng} \mathrm{mL} \mathrm{m}^{-1}$, we observed that $C_{\mathrm{CitH} 3}$ in the endotoxic mouse monotonically increased over time, rapidly reached a high level over $1 \mathrm{ng} \mathrm{mL^{-1 }}$ at $6 \mathrm{~h}$, and steadily increased to $1.385 \mathrm{ng} \mathrm{mL}^{-1}$ at $12 \mathrm{~h}$. The PCT concentration $\left(C_{P C T}\right)$ was as low as $0.075 \mathrm{ng} \mathrm{ml}^{-1}$ at $6 \mathrm{~h}$ and only increased to $0.078 \mathrm{ng} \mathrm{mL}^{-1}$ at $12 \mathrm{~h}(20$ times smaller than the $C_{\mathrm{CitH} 3}$ value). The IL-1 $\beta$ concentration $\left(C_{I L-1 \beta}\right)$ in the sample was $\approx 1.031 \mathrm{ng} \mathrm{ml}^{-1}$ at $3 \mathrm{~h}$ but finally decreased to $\approx 0.758 \mathrm{ng} \mathrm{mL}^{-1}$ at $12 \mathrm{~h}$ after repeating fluctuations. However, the $C_{\mathrm{CitH} 3}$ profile followed the endotoxic shock of the host consistently increasing from the initial point of LPS injection up to the endpoint of the animal life. As another approach of demonstration, we also successfully monitored the endotoxemia of another mouse model treated by YW3-56, an anticancer PAD inhibitor, via intraperitoneal injection (Figure 6b). As expected, our data showed that the treatment with YW3-56 significantly decreased $C_{\mathrm{CitH} 3}$ and $C_{\mathrm{IL}-1 \beta}$ as compared to those insulted only with LPS. Finally, we could monitor the dynamic response of the level of the biomarkers in such a shorter time interval which cannot be achieved by the current gold standard ELISA method.

Furthermore, we employed two different mouse models for the iNOBS assay: LPS-induced shock (LPSS) as an infectious model and hemorrhagic shock (HS) (30\% blood loss) as a noninfectious model, and replicated our recent finding that $\mathrm{CitH} 3$ is detected in LPSS but not in HS (Figure 6c,d). ${ }^{[8]}$ We measured the level of the target biomarkers in blood samples at $0,0.5$, and $12 \mathrm{~h}$ after LPS or HS insult (see Experimental Section). LPS led to an increase of $C_{\mathrm{CitH} 3}$ in the LPS-insulted mice from 0.022 to $1.137 \mathrm{ng} \mathrm{ml}^{-1}$ at 0.5 and $12 \mathrm{~h}$, respectively; while in the HS mice, very low levels of $C_{\mathrm{CitH} 3}$ (from 0.001 to $0.031 \mathrm{ng} \mathrm{ml}^{-1}$ ) were detected. The $C_{\mathrm{CitH} 3}$ levels at 0.5 and $12 \mathrm{~h}$ clearly distinguished the non-infectious and infectious systemic inflammation development with a high sensitivity. The $C_{I L-1 \beta}$ value also increased distinctively from 0.5 to $12 \mathrm{~h}$ after LPS insult. However, we observed in Figure $6 a$ that the IL- $1 \beta$ levels showed fluctuation over time. Therefore, IL-1 $\beta$ alone could hardly reflect the course of endotoxic shock. The concentration of PCT was too low to be sensitive for differentiating between LPS-induced endotoxic shock and hemorrhagic shock.

LPS is a central mediator that plays a critical role in the acute pathogenesis of endotoxemia and endotoxic shock. ${ }^{[7]}$ LPS can stimulate nuclear translocation of PAD and induce neutrophil extracellular trap (NET) formation. CitH3, as a component of the NETs, is released into circulation meanwhile. The temporal profile of the $\mathrm{CitH} 3$ biomarker measured over the lifetime of the endotoxic mouse model may well represent the timecourse development of the biological process responsible for CitH3 release from NETs into circulation. Generally, to provide timely and precise treatment of sepsis, it is critical to discriminate infectious inflammation from non-infectious inflammation at an early stage. However, they both represent alterations during common inflammatory stress responses and can be hardly differentiated well at an early state through conventional assay processes. ${ }^{[28]}$ The iNOBS data obtained for two different types of mouse models indicate that $\mathrm{CitH} 3$ measurement can 


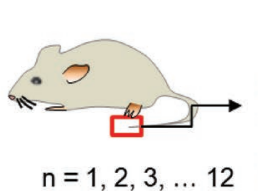

C

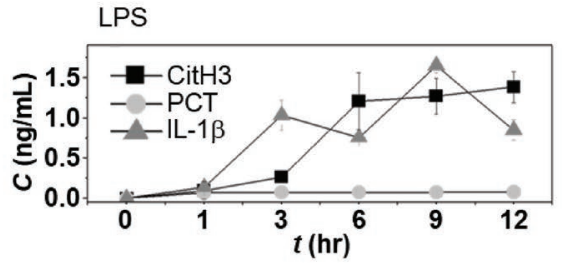

i) LPSS

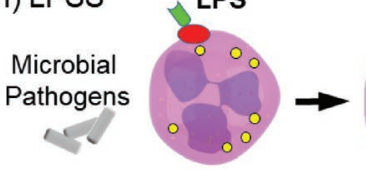

Neutrophil activation

d

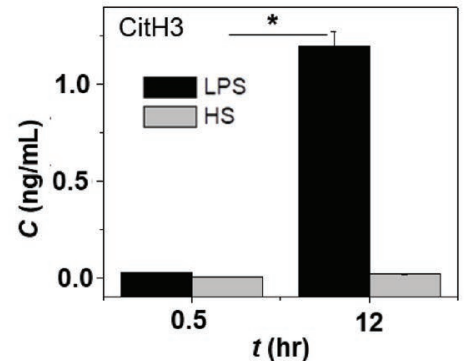

CitH3

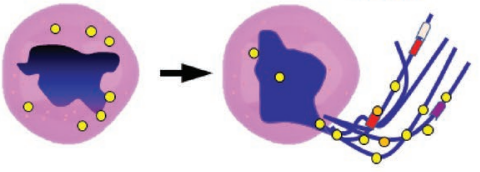

PAD4
Extracellular trap formation

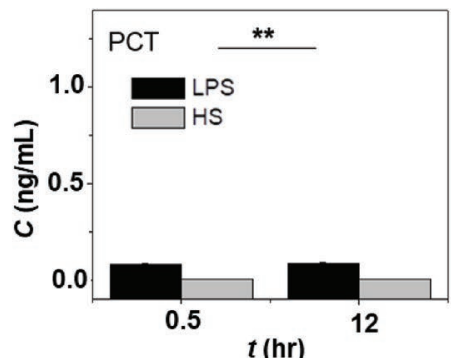

LPS-YW3-56

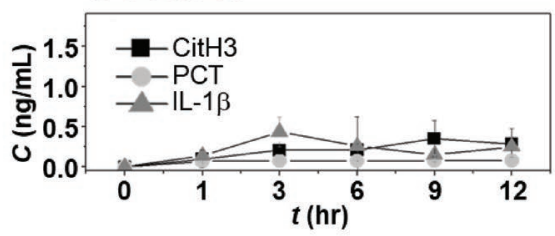

ii) HS

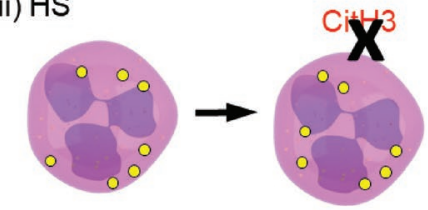

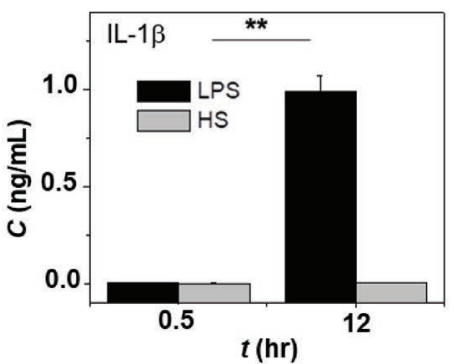

Figure 6. Multi-time-point $\mathrm{CitH} 3$ measurement for a living mouse with the iNOBS device. a) Time-course profiles of circulating CitH3, PCT, and IL-1 $\beta$ in a living mouse at $0,1,3,6,9$, and $12 \mathrm{~h}$ after LPS injection. At each measurement time point, a serum sample with a volume of $7.5 \mu \mathrm{L}$ was collected from the mouse tail. The values of the biomarker concentrations were extracted from the obtained calibration curve (Figure S8 and Table S5, Supporting Information). b) Time-course profiles of circulating CitH3, PCT, and IL-1 $\beta$ for a living LPS-injected mouse under treatment with YW3-56, an anticancer PAD inhibitor. c) Illustration of neutrophil behaviors in LPSS and HS models. CitH3 is a specific biomarker used to distinguish the LPS condition from the HS condition. d) $\mathrm{C}_{\mathrm{CitH} 3}, \mathrm{C}_{\mathrm{PCT}}$, and $\mathrm{C}_{\mathrm{IL}-1 \beta}$ measured for LPS and HS mouse models $(\mathrm{n}=20, * p<0.005$ and $* * p<0.05)$. In the test, the HS mouse was subjected to $30 \%$ blood loss without resuscitation, and its blood samples were collected at $0,0.5$, and $12 \mathrm{~h}$ after the HS procedure.

significantly distinguish between infectious and non-inflammatory responses..$^{[8,29]}$

\section{Conclusions}

Unlike cancer and other chronic diseases, infection-induced sepsis can rapidly and dynamically evolve. Management of severe sepsis imposes stringent requirements on diagnosis in terms of speed and sensitivity. ${ }^{[30]}$ In this study, we developed a miniaturized biosensor platform for detecting CitH3, a circulating sepsis biomarker released by neutrophils upon the first-line immune defense activity against infection. The biosensor monolithically integrated highly ordered, uniform, and packed self-assembled antibody-functionalized AuNH arrays and a highly photoresponsive few-layer $\mathrm{MoS}_{2}$ photoconductive channel into a small chip. We functionalized the AuNH arrays with a new high-affinity CitH3 monoclonal antibody developed by our lab. The unique combination of the nanoscale plasmonic structure, antibody, and two-dimensional (2D) material permited self-contained photoelectronic label-free detection of serum CitH3 with high speed, high sensitivity, high accuracy, and a large detection range. This study applied the remarkable performance of the iNOBS to demonstrate our capability of monitoring circulating $\mathrm{CitH} 3$ and other biomarkers in situ in a living mouse in the sepsis mouse model after LPS-induced endotoxic shock. From its biological basis and measured time-course profile, $\mathrm{CitH} 3$ is expected to provide a more accurate signature of infection-induced inflammation than PCT, which has been used in the conventional clinical diagnosis of sepsis. Recognizing the heterogeneity of septic conditions and inter-patient variability, there is a need for shifting clinical management of infections towards personalized treatment. ${ }^{[31,32]}$ The material processing used for the iNOBS construction is scalable for a fully standalone biosensor module for massively parallel analysis of multiple biomarkers at the point of care. The highly miniature iNOBS device may be eventually incorporated into a fully handheld portable POC diagnostic module without requring any bulky off-chip signal reader or instrument. This module would enable clinicians to monitor temporal biomarker profiles of patients in real-time and facilitate a biomarker-guided precision/personalized therapy of infections in a timely manner.

\section{Experimental Section}

Chemicals: 10-carboxy-1-decanethiol (C-10), Bovine serum albumin (BSA), Deoxyribonuclease (DNase), and Dimethyl sulfoxide (DMSO) 
were purchased from Sigma Aldrich. Polystyrene-b-poly (2-vinylpyridine) (PS-bP2VP) (S units: 213000; VP units: 153000) was purchased from Polymer Science. 1-ethyl-3-[3-dimethylaminopropyl] carbodiimide (EDC) and $\mathrm{N}$-hydroxysuccinimide (NHS) were purchased from Thermo Co. Ltd. IL-1 $\beta$ and the anti-IL-1 $\beta$ antibody were purchased from Life Technologies, Frederick, MD. PCT was purchased from ProSpec BiO and the anti-PCT antibody was purchased from Lifespan Biosciences. The high-affinity $\mathrm{CitH} 3$ monoclonal antibody was designed by us and generated in ProMab Biotechnologies, Inc. (Richmond, CA). YW3-56 was purchased from Cayman Chemical. Polydimethylsiloxane (PDMS) elastomer and curing agent were purchased from Corning. Nano-pure deionized (DI) water $(18.1 \mathrm{M} \Omega-\mathrm{cm})$ was produced in-house.

Animals: All animal experiments were conducted in compliance with the guidelines approved by the Animal Review Committee at the University of Michigan. Male C57BL/6) mice (9-10 weeks old) were purchased from the Jackson Laboratory (Bar Harbor, ME). Mice were housed for at least 3 days before any procedure.

Few-layer $\mathrm{MoS}_{2}$ photodetector channel fabrication: We fabricated A few-layer $\mathrm{MoS}_{2}$ photoconductive channel on a $\mathrm{SiO}_{2} / \mathrm{Si}$ substrate using a micro-printing method (Figure S3) (see Supporting information). ${ }^{[33]}$

Au nanoparticle Array Synthesis and Characterization: Arrayed AuNHs were fabricated as the surface parts of the Bio-NOF layer by controlling the surface energy using block copolymer (BCP) chemistry (see Supporting information).

LSPR spectroscopy and optical properties: The LSPR properties of gold nanoparticle-deposited surfaces were characterized by photo spectroscopy. To confirm the uniformity, LSPR spectral measurement was repeated for different surface locations of the Bio-NOF layer by automated positioning with a motorized stage. The LSPR sensitivity of the Bio-NOF layer to a bulk refractive index change was characterized by loading a water-glycol solvent mixture onto the AuNH array surface at different mixing ratios. The figure-of-merit (FOM) of the LSPR-based refractive index sensing was calculated for the Bio-NOF layer by dividing the refractive index (RI) unit by the full width at half maximum (FWHM) of the measured LSPR spectral curve.

Measurement Optoelectronic Performance: All the photocurrent measurements were performed using a semiconductor parameter analyzer (HP-4145B). Our measurements used a 650-nm laser light source (power density: $2.4 \mathrm{~mW}$ ). The baseline photocurrent signal of the device was calibrated. The power density of light transmitting through the Bio-NOF layer was measured using a power meter (Newport, 843-R).

Antibody Conjugation on Au Nanoparticle Array: The arrayed AuNHs were functionalized via self-assembled thiolate alkane 10-carboxy-1decanethiol (HS- $(\mathrm{CH} 2) 10-\mathrm{COOH})$ linkers. ${ }^{[16]}$ After a surface cleaning process, a mixture of $0.4 \mathrm{M} \mathrm{EDC}$ and $0.1 \mathrm{M} \mathrm{NHS}$ at a 1:1 volume ratio was loaded onto the functionalized AuNHs. Then, an antibody buffer solution was loaded and incubated to conjugate the AuNHs with anti$\mathrm{CitH} 3$ antibody molecules.

Integration of AuNH-deposited Bio-NOF Layer and Few-layer $\mathrm{MoS}_{2}$ Photoconductive Channel: After preparing the AuNH-deposited Bio-NOF layer and the atomically layered $\mathrm{MoS}_{2}$ photoconductive channel individually, we integrated them into a single on-chip device platform (see Supporting information).

Optoelectronic Biomarker Detection: The antibody-conjugated $\mathrm{AuNH}$ surfaces were treated with $10 \mu \mathrm{L}$ of $1 \%$ BSA in a $1 \times$ PBS blocking buffer to suppress the non-specific binding of background molecules in serum. The AuNH surfaces were thoroughly washed using $1 \times$ PBS to remove excessive reagents and background molecules. Then, a DNase-treated serum sample was loaded into the detection chamber. After washing processes, the photocurrent signal of the iNOBS device was measured in a detection chamber where temperature and pressure were well controlled.

Near-field Electromagnetic Radiation Calculation: To obtain near-field electromagnetic radiation around an $\mathrm{AuNH}$ structure, we solved the Helmholtz wave equation based on a finite element analysis method (FEA, COMSOL Multiphysics software) (see Supporting information).

Enzyme-linked Sandwich Assay (ELISA): ELISA is the current gold standard method to quantify $\mathrm{CitH} 3$ in a serum sample. A 96-well plate of
CitH3 ELISA kit was developed in our laboratory as described previously (see details in Supporting information). ${ }^{[8]}$

Western Blotting: Mouse serum was diluted with normal saline at 1:1 ratio $(15 \mu \mathrm{l}$ serum $+15 \mu \mathrm{l}$ normal saline) and added a $6 \mu \mathrm{L}$ buffer. The prepared solution was denatured and separated by sodium dodecyl sulfate-polyacrylamide gel electrophoresis (SDS-PAGE), and transferred to nitrocellulose membrane. The membrane was probed with an antiCitH3 polyclonal antibody (1:1000 diluted; ab5103, Abcam, Cambridge, MA, USA) and an anti-rabbit secondary antibody (1:3000 diluted; ab97051, Abcam, Cambridge, MA, USA). Ponceau S staining served as a control for equal loading.

Cecal Ligation and Puncture (CLP): Male mice (25-30 g) underwent the standardized CLP procedure as previously described. ${ }^{[4]}$ In our experiments, $75 \%$ of the cecum was ligated. After that, the cecum was perforated by a single puncture midway between the ligation and the tip of the cecum with a 27-G needle. After removing the needle, feces were extruded. The cecum was relocated, after that the fascia, abdominal musculature, and peritoneum was closed. The skin was also sutured. The sham mice were anesthetized and underwent laparotomy without a puncture or cecal ligation and served as the control.

Lipopolysaccharides (LPS)-induced Endotoxic Shock: LPS (35 mg kg-1) was injected into the mice intraperitoneally. Blood was collected at designated time points after LPS insult ( $n=3$ /time point). Mice without any insult or treatment served as normal controls ( $n=3$ /time point). For the treatment control, YW3-56 (5 mg kg-1 dissolved in DMSO vehicle) was intraperitoneally injected into mice after LPS.

Hemorrhagic Shock (HS): The procedure of hemorrhagic shock was performed as previously described. ${ }^{[8]}$ Briefly, male mice were anesthetized with $5 \%$ isoflurane and maintained with $2 \%$ isoflurane. With the heart rate and blood pressure being monitored by Ponemah Physiology Platform (Ground Instrument Systems, Valley View, $\mathrm{OH}$ ), the femoral artery of mice was cannulated with polyethylene 10 tubing (PE10, Clay Adams, Sparks, MD). Hemorrhagic shock with 30\% blood loss was accomplished as described previously. ${ }^{\left[{ }^{[8]}\right.}$ Animals from sham group received all procedures except cannulation and hemorrhage. The mice were sacrificed, and blood was collected at designated time points ( $n=3 /$ time point). Sham mice were performed the same procedures without being cannulated or subjected to hemorrhage ( $n=3 /$ time point). The serum samples were prepared by centrifuging the blood for 20 minutes at $2000 \times \mathrm{g}$.

Data Analysis: Results were presented as mean \pm standard deviation (SD). Differences among no less than 3 groups were assessed by one-way analysis of variance (ANOVA), followed by Bonferroni post hoc testing for multiple comparisons. Student's t-test was performed to determine the differences between the two groups.

\section{Supporting Information}

Supporting Information is available from the Wiley Online Library or from the author.

\section{Acknowledgements}

Y.P., B.R., and Q.D. contributed equally to this work. This research was supported by academic research fund at University of Michigan and the National Science Foundation (ECCS 1708706). Also, this work was funded by grants from Mcubed U049657 and Kickstart N022142 to Dr. Y. Li, and from UMHS-PUHSC Joint Institute U050150 to Dr. H. Alam.

\section{Conflict of Interest}

The authors declare no conflict of interest. 


\section{Keywords}

citrullinated histone $\mathrm{H} 3$ ( $\mathrm{CitH} 3$ ), localized surface plasmon resonance (LSPR), molybdenum disulfide (MoS2), optoelectronic biosensors, sepsis

Received: October 1, 2019

Revised: November 1, 2019

Published online: December 3, 2019

[1] F. B. Mayr, S. Yende, D. C. Angus, Virulence 2014, 5, 4.

[2] S. Laukemann, N. Kasper, P. Kulkarni, D. Steiner, A. C. Rast, A. Kutz, S. Felder, S. Haubitz, L. Faessler, A. Huber, C. A. Fux, B. Mueller, P. Schuetz, Medicine 2015, 94, e2264.

[3] B. D. W. Group, Clin. Pharmacol. Ther. 2001, 69, 89.

[4] J. C. Marshall, K. Reinhart, Crit. Care Med. 2009, 37, 2290.

[5] E. Kolaczkowska, P. Kubes, Nat. Rev. Immunol. 2013, 13, 159.

[6] I. Neeli, S. N. Khan, M. Radic, J. Immunol. 2008, 180, 1895.

[7] Y. Li, B. Liu, E. Y. Fukudome, J. Lu, W. Chong, G. Jin, Z. Liu, G. C. Velmahos, M. Demoya, D. R. King, H. B. Alam, Surgery 2011, $150,442$.

[8] B. Pan, H. B. Alam, W. Chong, J. Mobley, B. Liu, Q. Deng, Y. Liang, Y. Wang, E. Chen, T. Wang, M. Tewari, Y. Li, Sci. Rep. 2017, 7, 8972.

[9] J. N. Anker, W. P. Hall, O. Lyandres, N. C. Shah, J. Zhao, R. P. Van Duyne, Nat. Mater. 2008, 7, 442.

[10] B. Sepulveda, P. C. Angelome, L. M. Lechuga, L. M. Liz-Marzan, Nano Today 2009, 4, 244.

[11] X. H. Wang, T.-W. Chang, G. Lin, M. R. Gartia, G. L. Liu, Anal. Chem. 2017, 89, 611

[12] S. M. Russell, R. de la Rica, Sens. Actuators, B 2018, $270,327$.

[13] F. Yesilkoy, R. A. Terborg, J. Pello, A. A. Belushkin, Y. Jahani, V. Pruneri, H. Altug, Light: Sci. Appl. 2018, 7, 17152.

[14] K. M. Mayer, J. H. Hafner, Chem. Rev. 2011, 111, 3828.

[15] Y. Y. Wang, J. H. Zhou, J. H. Li, Small Methods 2017, 1, UNSP 1700197.

[16] P. Y. Chen, M. T. Chung, W. McHugh, R. Nidetz, Y. W. Li, J. P. Fu, T. T. Cornell, T. P. Shanley, K. Kurabayashi, ACS Nano 2015, 9, 4173.

[17] Y. Zhang, Y. Tang, Y.-H. Hsieh, C.-Y. Hsu, J. Xi, K.-J. Lin, X. Jiang, Lab Chip 2012, 12, 3012.
[18] Y. Park, B. Ryu, B. R. Oh, Y. Song, X. Liang, K. Kurabayashi, ACS Nano 2017, 11, 5697.

[19] M. Chen, H. Nam, H. Rokni, S. Wi, J. S. Yoon, P. Chen, K. Kurabayashi, W. Lu, X. Liang, ACS Nano 2015, 9, 8773.

[20] Q. Deng, B. Pan, H. Alam, Y. Liang, B. Liu, N. Mor-Vaknin, X. Duan, A. M. Williams, Y. Tian, Z. Wu, J. Zhang, Y. Li, Front. Immunol. 2019, Under review.

[21] J. Sebaugh, P. McCray, Pharmaceutical Statistics 2003, 2, 167.

[22] S. J. Parker, P. E. Watkins, Br. J. Surg. 2001, 88, 22.

[23] K. A. Wichterman, A. E. Baue, I. H. Chaudry, J. Surg. Res. 1980, 29, 189.

[24] S. Q. Latifi, M. A. O'Riordan, A. D. Levine, Infect. Immun. 2002, 70, 4441.

[25] D. G. Remick, G. R. Bolgos, J. Siddiqui, J. Shin, J. A. Nemzek, Shock 2002, 17, 463.

[26] K. Doi, A. Leelahavanichkul, P. S. Yuen, R. A. Star, J. Clin. Invest. 2009, 119, 2868.

[27] S. Ruiz, F. Vardon-Bounes, V. Merlet-Dupuy, J.-M. Conil, M. Buléon, O. Fourcade, I. Tack, V. Minville, Intensive Care Medicine Experimental 2016, 4, 22.

[28] W. Xiao, M. N. Mindrinos, J. Seok, J. Cuschieri, A. G. Cuenca, H. Gao, D. L. Hayden, L. Hennessy, E. E. Moore, J. P. Minei, P. E. Bankey, J. L. Johnson, J. Sperry, A. B. Nathens, T. R. Billiar, M. A. West, B. H. Brownstein, P. H. Mason, H. V. Baker, C. C. Finnerty, M. G. Jeschke, M. C. Lopez, M. B. Klein, R. L. Gamelli, N. S. Gibran, B. Arnoldo, W. Xu, Y. Zhang, S. E. Calvano, G. P. McDonald-Smith, D. A. Schoenfeld, J. D. Storey, J. P. Cobb, H. S. Warren, L. L. Moldawer, D. N. Herndon, S. F. Lowry, R. V. Maier, R. W. Davis, R. G. Tompkins, Inflammation, P. Host Response to Injury Large-Scale Collaborative Research, J. Exp. Med. 2011, 208, 2581.

[29] T. Zhao, B. Pan, H. B. Alam, B. Liu, R. T. Bronson, Q. Deng, E. Wu, Y. Li, Sci. Rep. 2016, 6, 36696.

[30] P. Y. Chen, N. T. Huang, M. T. Chung, T. T. Cornell, K. Kurabayashi, Adv. Drug Delivery Rev. 2015, 95, 90.

[31] R. Satija, A. K. Shalek, Trends Immunol. 2014, 35, 219.

[32] J. W. Uhr, Science 1964, 145, 457.

[33] H. Nam, S. Wi, H. Rokni, M. Chen, G. Priessnitz, W. Lu, X. Liang, ACS Nano 2013, 7, 5870.

[34] T. Zhao, Y. Li, B. Liu, Z. Liu, W. Chong, X. Duan, D. K. Deperalta, G. C. Velmahos, H. B. Alam, Surgery 2013, 154, 206. 